\title{
Práctica profesional en contexto de pandemia: algunas reflexiones sobre el sistema educativo chileno
}

\section{Professional Practice in the Context of a Pandemic: Some Reflections on the Chilean Education System}

\author{
Jorge Pizarro-Vargas ${ }^{1}$
}

\begin{abstract}
RESUMEN
El contexto actual, en Chile y el mundo, ha implicado la realización de una práctica profesional pedagógica anormal. Cabe reflexionar sobre los procesos de enseñanza-aprendizaje y las metodologías que se están aplicando en el sistema educativo chileno en este contexto, donde las clases -de emergencia- a distancia se han convertido en la única forma de mantener el contacto entre la escuela y las/os estudiantes. A partir de la propia experiencia de práctica profesional en contexto de pandemia, tomando en cuenta determinados procesos y metodologías, el presente artículo ofrece reflexiones con respecto al sistema educativo chileno y a cómo estamos entendiendo el entramado en el cual nos insertamos las/os docentes y las/os estudiantes.
\end{abstract}

Palabras clave: práctica profesional pedagógica; educación de emergencia; educación a distancia; Lenguaje y Comunicación; intervención didáctica; COVID-19; pandemia.

\begin{abstract}
The current context, in Chile and the world, has implied the realization of an abnormal pedagogical professional practice. It is worth reflecting on the teaching-learning processes and the methodologies that are being applied in the Chilean educational system in this context, where remote-emergency-classes have become the only way to maintain contact between the school and the students. students. From own experience of professional practice in the context of a pandemic, taking into account certain processes and methodologies, this article offers reflections regarding the Chilean educational system and how we are understanding the framework in which teachers and students are inserted.
\end{abstract}

\footnotetext{
${ }^{1}$ Licenciado en Educación y Profesor de Enseñanza Media de Lenguaje y Comunicación, Universidad Academia de Humanismo Cristiano, Santiago, Chile; estudiante de Magíster en Comunicación, Universidad Austral de Chile, Valdivia, Chile; profesorjorgepizarrovargas@ gmail.com; https://orcid.org/0000-0003-0700-9772.
} 
Keywords: Pedagogical professional practice; emergency education; long distance education; Language and Communication; didactic intervention; COVID-19; pandemic.

En medio del miedo y de la depresión que prevalecen en este tiempo va surgiendo, por debajo imperceptiblemente, atisbos de otra manera de vivir que busca, al borde del abismo, la recuperación de una humanidad que se siente a sí misma desfallecer. (Sábato, 1998, p.9)

Desde que decidí estudiar el Programa de Formación Pedagógica en Enseñanza Media de la Universidad Academia de Humanismo Cristiano, me han preguntado en una innumerable cantidad de ocasiones por qué decidí estudiar pedagogía. Dar una respuesta acertada o, si se quiere, “políticamente correcta”, se transformó casi en una necesidad. La verdad es que no sabría decir si existe una respuesta correcta a esa pregunta. Hay respuestas basadas en la frase hecha o en el lugar común, en las que no indagaré aquí, pero me gustaría pensar que en la labor docente hay una doble satisfacción: por un lado, la satisfacción personal de dedicarnos a algo que nos gusta y, por otro lado, aquello que tiene que ver con la transformación de la vida de las y los estudiantes, en la búsqueda incesante por convivir en una sociedad más justa. Estoy convencido de que la educación cumple un rol importantísimo en el asentamiento de las bases necesarias para convivir en sociedad. Y, a partir de ahí, cabe preguntarse: ¿qué es la educación?

En 1977 circuló por los colegios argentinos un cuadernillo publicado y distribuido por el Ministerio de Cultura y Educación de la dictadura argentina, titulado Subversión en el ámbito educativo (conozcamos a nuestro enemigo), en el cual se daban directrices acerca del supuesto funcionamiento de movimientos marxistas o comunistas en las organizaciones estudiantiles que se debían identificar para evitar su propagación. En la presentación del cuadernillo se escribe acerca de la función específica de las y los docentes en esa construcción de mundo impulsada por la dictadura y, en ese sentido, resulta incluso paradójico que dicha construcción parezca estar muy 
cercana a lo que estamos entendiendo hoy como acción docente, pero relacionada con el reconocimiento del supuesto accionar marxista:

Esa acción docente, por ser tal, debe comprender mucho más que la simple transmisión de conocimientos, como se dijera; ha de contribuir a la formación de quienes mañana, con solidez de principios, tomarán la antorcha que recibimos de nuestros padres y para que ello sea posible es menester estar preparados. (Ministerio de Cultura y Educación, 1977, p.6)

Según Federico Lorenz (2003), el cuadernillo perseguía evidentemente reprimir cualquier tipo de organización estudiantil incipiente, sobre todo después de la detención, tortura y desaparición de algunos dirigentes estudiantiles el año anterior, quienes habían participado en una protesta por el boleto escolar, denominada históricamente como "La noche de los lápices".

Abraham Magendzo (2003), en un artículo llamado "Pedagogía crítica y educación en derechos humanos", establece que la pedagogía que se transforma en un obstáculo para ejercer la autosuficiencia y la libertad de las personas se convierte inmediatamente en un sistema represor. Se entiende que las y los docentes son en parte responsables de aquellos saberes, conocimientos y aprendizajes que son transmitidos, motivados y entregados a las y los estudiantes. Se podría decir que aquello es casi un mandato necesario para cualquier sistema educativo que se considere a sí mismo un sistema democrático, reflexivo, formador de sujetos críticos y participantes de la sociedad. En este caso, el cuadernillo Subversión en el ámbito educativo (conozcamos a nuestro enemigo) utiliza discursos de progreso para, justamente, evitar la posibilidad de la formación de estos mencionados sujetos. Así, dicho cuadernillo persigue objetivos relacionados con mecanismos de control social, más que objetivos de libertad pedagógica. El discurso utilizado tiene que ver con el rol de las y los docentes y se plantea desde la dictadura para ejercer el poder en el ámbito educativo. A partir de aquí, se podrían establecer cuestiones relativas al rol de las y los docentes, la importancia de la transmisión de la historia reciente o la reflexión acerca de ciertas realidades culturales relacionadas con el control social de manera violenta, pues se entiende que la memoria es fundamental para los procesos reflexivos de las y los estudiantes. Finalmente, todo eso es la educación. Y en ese sistema nos insertaremos las y los -por ahora- aspirantes a profesoras y profesores. 
El presente artículo recoge y reflexiona sobre la práctica profesional que llevé a cabo durante el primer semestre del año 2020 y espero que funcione fundamentalmente como una invitación, para todas las actoras y actores sociales, a reflexionar con respecto a qué estamos entendiendo por educación. También es una invitación a la autoconciencia de la coerción escondida, a materializar acciones liberadoras y a lo que idealmente algunos creemos que debe ser la educación, no solo desde la reflexión académica, sino principalmente desde nuestra incidencia en la vida de las y los estudiantes y en las políticas públicas actuales que apuntan a la privatización del conocimiento y, finalmente, a la privatización de nuestras vidas.

\section{Las condiciones de desarrollo de la práctica profesional}

Para comenzar, es absolutamente necesario que, cual noticiero de circulación masiva y de financiamiento empresarial, me refiera a las condiciones determinadas por la pandemia de COVID-19 en las que se desarrolló el proceso de práctica profesional: la educación -de emergencia- a distancia.

El contexto nacional y mundial ha condicionado el proceso de práctica a la realización de clases virtuales, lo cual necesariamente ha influido en las estrategias de enseñanza-aprendizaje y en las metodologías utilizadas: no estábamos preparados ni preparadas. Lo anterior no se refiere solamente a los procesos de prácticas profesionales, evidentemente, sino que a todo un sistema educacional que no estaba destinado a desarrollarse de manera virtual y a distancia.

Estas condiciones determinaron no solo la cantidad de horas a cumplir para completar el proceso de práctica profesional, sino también la forma en la que esta se realizó, al menos en mi caso: mi función fue fundamentalmente la de apoyar a la profesora guía del colegio, de manera que nos distribuimos el trabajo de un octavo año y un primero medio en los que la profesora trabaja. Por otro lado, tomé como profesor titular las clases de un tercero medio, también a cargo de la profesora guía. Es necesario mencionar que, en términos de organización del colegio, el octavo año es solo un curso, en cambio, el primero y el tercero medio constituyen dos cursos por nivel, pero las clases sincrónicas online se hacen para ambos cursos. Es decir, las horas de trabajo sincrónico con el primero medio están destinadas a ambos cursos y así también sucedió con el tercero medio. 
A partir de lo anterior, uno de los principales problemas que enfrenté tuvo que ver con la información disponible respecto a la educación -de emergencia- a distancia. No existía información acabada, probada y validada con respecto a la eficacia de nuestras metodologías aprendidas en el contexto de la educación -de emergencia- a distancia. Lo anterior ha implicado que los y las practicantes nos hemos movido un poco a ciegas, a partir de muchísima intuición y con altos grados de predisposición, pero sin tener claro si las acciones emprendidas y las metodologías aplicadas son efectivas.

Otro problema tuvo que ver con un tema fundamental en el proceso de práctica: la transferencia efectiva de horas presenciales a virtuales. La asignatura de Lenguaje y Comunicación (Lengua y Literatura, según los nuevos lineamientos curriculares ministeriales) tiene un valor importante en la carga de horas semanales en el sistema educacional chileno. Lo anterior quiere decir que ese valor, en términos de cantidad de horas lectivas, se manifiesta supuestamente a nivel social. Así también se manifiesta en términos de pruebas estandarizadas de medición de calidad educativa y pruebas de selección universitaria. Lo concreto es que las disposiciones ministeriales indican que las y los estudiantes tienen más horas lectivas de Lenguaje y Comunicación que de otras materias. En el establecimiento en el que realicé la práctica, las horas pedagógicas asignadas por semana en contexto de presencialidad se transfirieron a solo dos horas pedagógicas virtuales. Considerando que las horas presenciales por semana eran siete para octavo básico, ocho para primero medio y cinco para tercero medio, es evidente que se vuelve imposible abarcar todos los contenidos inicialmente estipulados en solo dos horas de clases virtuales sincrónicas por nivel. Al ser una cuestión evidente desde el punto de vista práctico, durante el primer semestre de 2020, el Ministerio de Educación publicó un documento denominado "Priorización Curricular", el cual contiene los lineamientos curriculares indicados en el contexto de la pandemia por COVID-19. Así las cosas, se debe entonces esperar que la transferencia de horas planteada resguarde la cobertura curricular y, al mismo tiempo, ofrezca a las y los estudiantes las condiciones necesarias para la correcta adquisición de saberes.

Además, el contexto de pandemia que estamos viviendo ha condicionado la metodología utilizada para los procesos de enseñanza-aprendizaje. En lo que a mí respecta, la mayor parte de las clases que intento planificar consisten en conversaciones, debates y discusiones, con respecto a los diferentes discursos que planteo como temáticas guía de las dinámicas de aula. La idea ha 
sido siempre motivar a las y los estudiantes para que respondan a una batería de preguntas diversas y que, además, contesten a una pregunta base: “¿por qué?”. Lo anterior tiene que ver, evidentemente, con que aquello que busco es que las y los estudiantes sean capaces de argumentar oralmente sus propias afirmaciones. La idea fundamental es que se produzca, de alguna manera, una especie de monólogo argumentativo (Dolz, 1994) acerca de la temática que se trata en clases y, a partir de ahí, un debate (discurso interactivo) entre las y los estudiantes.

La realidad es que durante las clases virtuales las y los estudiantes no suelen activar sus cámaras ni sus micrófonos (salvo excepciones). La clase entonces se convierte en una presentación por parte del docente de los temas tratados y la lectura de las opiniones de las y los estudiantes, expresadas en el "chat" de la plataforma virtual. En esas condiciones es muy difícil o casi imposible que se materialice el discurso interactivo o el monólogo argumentativo, pues básicamente lo que sucede es que las y los estudiantes no hablan, pues no se sienten partícipes de la dinámica de la clase. Dicho de otra manera, se hacía muy difícil o casi imposible, en la realidad en la que yo estaba inserto, generar una dinámica de clase de discusión interactiva.

\section{La intervención didáctica}

\section{Contexto}

La metodología utilizada por el establecimiento para la enseñanza -de emergencia- a distancia en la asignatura de Lenguaje y Comunicación está relacionada con el desarrollo de ciertas habilidades de lectura de las y los estudiantes, a través de ejercicios de modelamiento de preguntas y respuestas con respecto a un texto.

¿Cuáles son los objetivos que persigue la fundación a la cual pertenece el colegio al utilizar la metodología de desarrollo de habilidades de lectura? Lo primero que se puede identificar es que para la fundación administradora resulta fundamental tener éxito en mediciones estandarizadas como el Simce o la prueba de selección universitaria. A las y los estudiantes prácticamente se les entrena para responder preguntas de comprensión de lectura que son típicas de estas pruebas. Las clases a distancia están destinadas al modelamiento de las respuestas que puedan entregar las y los estudiantes en todos los niveles. Las clases para primero y tercero medio están alojadas en una 
carpeta de Google Drive del colegio y lo único que la o el docente debe hacer es extraerlas y aplicarlas. Es decir, la o el docente es ejecutora o ejecutor de una clase que planificó otra u otro docente que ni siquiera pertenece al mismo colegio, sino a otro de los establecimientos administrados por la fundación. A las docentes de Lenguaje y Comunicación de la sede en la que realicé la práctica profesional les toca planificar octavo año y son algunas de estas planificaciones en las que he podido incidir. Dichas planificaciones circularán, además, en los otros colegios administrados por la fundación.

En términos prácticos, las dos horas pedagógicas semanales destinadas a Lenguaje y Comunicación se dividen en dos clases, realizadas en días y horarios distintos. La primera clase semanal es obligatoriamente una clase virtual sincrónica, por medio de una reunión en una plataforma de comunicación en línea, en la que se hace una presentación a las y los estudiantes donde se incluye la presentación del objetivo, la explicación de la habilidad de lectura destinada a esa clase (inferir, interpretar, explicar, etc.) y el modelamiento de una respuesta tipo para una pregunta predeterminada. Además, se encarga a las y los estudiantes una tarea denominada "práctica independiente" que consiste en responder una o dos preguntas con respecto a un texto, siguiendo las instrucciones de modelamiento hechas en la clase. La segunda clase puede ser sincrónica, por medio de una reunión en la plataforma antedicha, o asincrónica, alojando en la misma plataforma el solucionario de la "práctica independiente" encargada en la primera clase.

En ese contexto y con el objetivo de ofrecer a las y los estudiantes de tercero medio (nivel del que me hice cargo como profesor titular) la posibilidad de hacer lecturas más libres de una batería de textos determinada, decidí proponer al colegio una actividad paralela para la asignatura, en la que planifiqué cinco clases relacionadas con el tema de la pandemia y cómo se han enfrentado de manera personal algunas cuestiones propias de la realidad. Para lo anterior, seleccioné algunos textos literarios y no literarios que ayudaran a guiar la reflexión libre con respecto al tema planteado. Además, la propuesta de actividad paralela persiguió un objetivo que no solo tuvo que ver con las estrategias de lectura de las y los estudiantes, sino también con su predisposición al enfrentar la tarea de la lectura. Durante las primeras dos semanas de realización de las clases indicadas por el colegio, pude identificar que la predisposición de las y los estudiantes a la lectura presentaba un nivel de motivación bajísimo: básicamente no les apetecía leer ni realizar sus prácticas independientes. En ese contexto y con el propósito de elevar los niveles de motivación 
hacia la lectura, la propuesta de actividad paralela se constituyó como un intento por llevar a las y los estudiantes a otro plano de la lectura: el goce literario y la relación con sus experiencias de vida. En mi propia experiencia, establecí una relación personal con los discursos y relatos que comencé a leer durante mi adolescencia y, en términos de relación íntima, me parece que las y los docentes deben necesariamente transmitir de alguna manera aquellos discursos y relatos que pueden entregar luces para la reflexión de las y los estudiantes. Dichos discursos y relatos, además, ofrecen puntos de vista alternativos a los discursos oficiales o hegemónicos, permitiendo observar las cosas desde distintas perspectivas.

En esta actividad paralela pude, de alguna manera, transmitir aquello que quería transmitir, lo que finalmente tiene que ver con lo que entiendo que debería ser el rol docente en su relación disciplinar con las y los estudiantes. Así, he diferenciado aquí esas dos situaciones de enseñanza descritas, las clases encargadas e indicadas por el colegio y la propuesta de una actividad alternativa paralela a dichas clases, de manera de poder hacer primero un análisis por separado para luego, en las reflexiones y en las conclusiones, referirme de forma transversal a ambos procesos. Por un lado, entonces, estará la “propuesta de intervención didáctica” y, por otro lado, las "clases oficiales".

\section{Reflexiones sobre las unidades}

A lo largo de este documento he mencionado algunas cuestiones que tienen que ver con la metodología utilizada por el establecimiento en la asignatura de Lenguaje y Comunicación. Dicha metodología persigue objetivos relacionados con posibles resultados en pruebas de medición estandarizadas, incluyendo la prueba de selección universitaria. En ese contexto, es interesante mencionar algunas dinámicas de clases que se producen en el formato que el colegio o la fundación ha aplicado en contexto de clases -de emergencia- a distancia. Según tengo entendido, dicho formato también ha sido aplicado en contexto de presencialidad.

Clases oficiales. Da la impresión de que el formato por el cual opta el colegio, en términos de metodologías y estrategias de enseñanza-aprendizaje, está ya estandarizado. Es más, las presentaciones en Power Point siguen todas un mismo modelo. Este último tiene que ver con el modelamiento de estrategias lectoras que no buscan, en absoluto, ninguna formación desde la 
pedagogía crítica ni desde la educación formadora de sujetos críticas y críticos. Lo anterior evidencia una forma de entender la educación como un entrenamiento y una aplicación de estrategias que seguramente implicarán el éxito para las y los estudiantes. Es interesante analizar el rol de el o la docente en este tejido educativo, pues parece ser que la habilidad principal de una profesora o profesor está reducida a la capacidad de ejecutar una planificación tras otra, aplicando el modelo una y otra vez. A partir de lo que señala Rockwell (1997) con respecto a las prácticas cotidianas, podríamos decir que el proceso en el que las y los estudiantes interiorizan saberes se encuentra absolutamente restringido a la capacidad que tiene un sujeto de entrenar habilidades determinadas. En estas prácticas no se produce la apropiación de conocimientos diversos, valores ni formas de vivir y, por tanto, aquello que señala Rockwell con respecto a la escuela cotidiana es un imposible: “A partir de esas prácticas los alumnos se apropian diversos conocimientos, valores, formas de vivir y de sobrevivir. La experiencia formativa es importante también para los maestros" (Rockwell, 1997, p.14).

En línea con lo anterior, es interesante preguntarse qué es lo que sucede con colegios que pertenecen a ciertas fundaciones $\mathrm{y}$, finalmente, con la educación particular y particularsubvencionada en este país. Evidentemente, parece ser que las razones económico-administrativas de las instituciones escolares se ponen por sobre el sentido pedagógico de las mismas, lo cual parece representar un retroceso en términos de políticas educacionales, pues solo estaríamos convirtiendo a los colegios en fábricas de fuerza de trabajo, en productores de seres humanos que desarrollan habilidades suficientes para entender qué es lo que deben hacer, pero sin considerar un proceso reflexivo que lleve a ese ser humano a pensar su vida, su comunidad, su realidad. Además, habría que preguntarse también qué entienden estas fundaciones con respecto al concepto de éxito de las y los estudiantes que ahí acuden. El éxito parece estar relacionado a la consecución de ciertos resultados en pruebas de medición estandarizadas y en pruebas de selección académica, y abarcar solo una dimensión de las y los seres humanos: aquella que tiene que ver con el desarrollo de ciertas tareas determinadas y encomendadas, a cambio de un refuerzo positivo o una recompensa. Dichos conceptos tienen que ver evidentemente con la idea de mercantilización del sistema educativo y cómo en ese panorama se han desarrollado pruebas como el Simce, que implican necesariamente la validación del sistema: "En resumen, desde la perspectiva de las escuelas, ambas presiones, del mercado y el Estado evaluador, coinciden en incentivar la implementación de 
mecanismos, primero, de selección social y, luego, de preparación para el Simce, y ambos propósitos son plenamente convergentes." (Bellei, 2020, p.89).

Intervención didáctica. Como he mencionado, el objetivo principal de la intervención didáctica realizada tuvo que ver con las reflexiones respecto a cómo enfrentan la pandemia las y los estudiantes de tercero medio, en términos de confinamiento y clases -de emergencia- a distancia. Es así como propuse una serie de artefactos literarios, cinematográficos y fotográficos que motivaran dichos procesos reflexivos. Además, planteé la posibilidad de una actividad discursiva que, de alguna manera, ayudaría a las y los estudiantes en el proceso formativo a distancia, pues se presentaba como una actividad alternativa a sus clases oficiales. Básicamente, propuse una conversación que implicara tres ámbitos de nuestra vida: los artefactos -artísticos o no artísticos-, la convivencia en comunidad y las experiencias personales, como detonadores de ciertos procesos reflexivos. Además, dado que las y los estudiantes aludidas y aludidos pertenecen al nivel de tercero medio, en términos de estrategias de enseñanza-aprendizaje, me pareció necesario el desarrollo de relaciones intertextuales, no solo porque aparece en el currículum, sino porque creo que efectivamente es uno de los aspectos importantes en los procesos de formación en la disciplina de Lenguaje y Comunicación. La intertextualidad ha sido un trabajo que no se ha hecho evidente, pero espero que haya tenido sentido para las y los estudiantes, y haya contribuido a su proceso formativo. Al mismo tiempo, en ningún caso esta intervención obstaculizó el desarrollo de sus clases oficiales, sino más bien ayudó a llenar ese vacío que, en términos formativos, me parece que existe en colegios donde la metodología se resume a la aplicación de modelajes de habilidades.

Respecto a lo que aquí he denominado como intertextualidad y discurso interactivo, la idea fue mostrar diferentes discursos o relatos sobre temas relacionados con la pandemia y guiar la reflexión hacia las habilidades de los y las estudiantes para establecer relaciones, diálogos o discusiones entre ellas y ellos. Además, se buscó que fuesen capaces de establecer relaciones no solo entre los diferentes artefactos vistos, escuchados o leídos, sino también respecto a situaciones actuales en las que ellas y ellos viven inmersas/os. Con este propósito se incluyeron manifestaciones artísticas y culturales que les fuesen cercanas en el tiempo y en el estilo. Lo último se justifica en aquello que señala Carlos Scolari (2014) con respecto a lo que él llama "narrativas transmedia": "una parte de los receptores no se limita a consumir el producto cultural, sino que se embarca en la tarea de ampliar el mundo narrativo con nuevas piezas textuales" (Scolari, 2014, 
p.72). Por este motivo, se incluyeron manifestaciones artísticas de músicos actuales, con el fin de que las y los estudiantes pudieran entrar a las temáticas a partir de una realidad cercana y, desde ahí, establecer conexiones entre sus realidades, sus experiencias y la pandemia.

Como último objetivo, me planteé la posibilidad de establecer conversaciones más informales que las que normalmente ellas y ellos tienen en clases. Lo anterior no con el objeto de ganarme su simpatía, sino que para manifestar durante las conversaciones aquellas reflexiones a las que he hecho alusión. Mi observación inicial fue que las y los estudiantes del tercero medio funcionaban como entrenados/as para recibir un estilo de clase que no cautivaba su interés. Sus manifestaciones de desagrado, desánimo y desgana fueron abundantes en términos de cómo enfrentaron la clase de Lenguaje y Comunicación. No estoy sosteniendo que todas las clases deben contar con un atractivo del tipo "entretenimiento", pues estaría reduciendo el rol de las y los docentes al de presentadoras/es, como he mencionado al inicio del documento. Sin embargo, la informalidad y la cotidianeidad de las clases creo que ayudaron a que, al enfrentar las lecturas, los cortometrajes, la música o las fotografías que incluí en la intervención, se percibiera un halo de multiplicidad de lecturas posibles, sin necesariamente perseguir el objetivo propio de las clases oficiales.

\section{Análisis y reflexión de situaciones de enseñanza-aprendizaje}

Se puede establecer que el llamado análisis crítico del discurso busca dar cuenta de las representaciones del mundo y de las relaciones sociales originadas en los discursos. A partir de esta consideración, para mí fue importante que junto a las y los estudiantes de tercero medio pudiéramos establecer una relación en la que se manifestaran sus propios discursos. Para Fairclough (2003), el análisis crítico del discurso tiene su raíz en la semiosis, entendida ella como cualquier forma de creación de significados, entre las que se encuentra (instalada quizás como la más importante) el lenguaje. La semiosis sería parte irreducible de los diversos procesos sociales y, si se entiende la vida como una compleja red de prácticas sociales, el análisis crítico del discurso sería una herramienta que permite vincular la estructura a la acción, en el sentido de que permite relacionar las maneras de construir discursos con elementos pertenecientes a determinadas prácticas sociales y a sus representaciones. Los discursos son representaciones de una determinada vida social: 
Los actores sociales de distinta posición 'ven' y representan la vida social de maneras distintas, con discursos diferentes. Por ejemplo, la vida de los pobres y de los desfavorecidos se representa mediante discursos diferentes en las prácticas sociales del gobierno, de la política, de la medicina y de las ciencias sociales. (Fairclough, 2003, p.182)

En líneas muy generales, existen tres tipos de procesos básicos que una o un hablante puede manifestar para referirse al mundo, es decir, para representarlo. Los primeros son los llamados "procesos materiales", que son aquellos que expresan acciones o acontecimientos de la realidad tangible y que disponen y ordenan la esfera de la producción. Estos procesos siempre implican, como función propia, la contribución activa de un actor o instrumento. Los segundos son los “procesos mentales", es decir, aquellas manifestaciones que están relacionadas con la conciencia. Estos se manifiestan, por ejemplo, mediante verbos de percepción como ver, oír o sentir, verbos de cognición como saber o conocer, verbos de reacción como agradar, odiar o gustar, entre otros. Por último, están los “procesos de relación”, los cuales solo ponen en relación dos roles. Hay tres modos de marcar la relación: mediante la atribución o caracterización, mediante la identificación y mediante la posesión. Era imposibles reconocer todos estos procesos por medio de las clases oficiales del establecimiento educacional, pues, como ya se ha dicho, estas solo se limitaban a modelar cierto tipo de habilidad lectora entendida como correcta. Lo más importante para mí es que, con la intervención didáctica, creo que las y los estudiantes se sintieron libres para emitir sus opiniones, utilizar sus procesos materiales y mentales, y procesar la información de manera de manifestar su rol de estudiante. Al mismo tiempo, creo que la batería de textos -literarios y no literarios- implicó lecturas que, en términos de cantidad, requerían sistematicidad y en algún punto busqué dicho objetivo.

Es importante tener en cuenta, sin embargo, que en la escuela no se dan procesos de reproducción, de relaciones sociales y de poder; se dan además procesos de resistencia y de lucha, así como de apropiación de la cultura, que son parte esencial de la trama social cotidiana. La simultaneidad de estos procesos hace posible la transformación histórica de la institución escolar. (Rockwell, 1997, p. 56)

Creo que las y los estudiantes firmaron el pacto pedagógico de la intervención didáctica, en la medida que establecimos una relación más cercana con los temas propuestos y las lecturas sugeridas, a partir de aquella “conversación de los viernes". Una de las cuestiones principales a las 
que he hecho alusión ha sido la motivación con la cual enfrentaban la lectura. Si bien no puedo afirmar que las y los estudiantes del tercero medio son hoy en día más lectores y lectoras que antes (ni siquiera sé si ese debería ser un objetivo pedagógico), lo que sí puedo asegurar es que al enfrentar las lecturas y los artefactos que yo les entregué, se materializó una conversación más libre con respecto a las posibles lecturas de dichos artefactos. Es decir, su relación con la literatura y los demás artefactos se convirtió necesariamente en una cuestión de experiencia estética, más relacionada con lo emocional o la apreciación que con la obligación de responder correctamente una pregunta determinada (que, además, ha estado consistentemente mal construida).

Una última reflexión se refiere a la actividad práctica propuesta por mí para ser desarrollada. Dicha actividad persiguió el objetivo de impulsar la reflexión y, al hacerlo, materializarla en términos textuales. Lo que les propuse a las y los estudiantes tenía que ver con la escritura de una frase simple en la que ellas y ellos definieran lo que ha significado la pandemia en sus vidas o en las de las personas a su alrededor. Después de la construcción de la frase, debían enviarme un vídeo con la misma, en los términos que ellas y ellos quisieran. La verdad es que mi idea era que tomaran decisiones, hicieran uso de su autonomía y construyeran una idea simple resumible en una frase. Recibí algunos videos de las y los estudiantes, y parece ser que la capacidad de síntesis no es una habilidad desarrollada por ellas y ellos. La mayoría construyó muchísimo más que una frase simple. Por otro lado, la expresión de lo que creen y sienten me parece que ha sido la tónica principal de sus frases y vídeos y, por esta razón, creo que se puede apelar a la expresión más que a otras habilidades. En términos de toma de decisiones, constantemente me encontré explicando la actividad una y otra vez y, como no era mi idea entregarles la forma en la que ellas y ellos deben hacer las cosas, intenté guiarlas y guiarlos de manera que tomaran decisiones propias, sin la mediación del profesor.

\section{Conclusiones}

Me parece que es interesante levantar la discusión con respecto al sentido de la educación en todas y cada una de las intervenciones que uno realice con estudiantes. No quiero decir aquí que se tenga que conversar todas las clases con ellos o ellas sobre cuál es el sentido de educarnos o por qué debe existir un sistema educacional determinado. Más bien me refiero a instalar el debate con 
respecto a cuestiones importantes relacionadas con la educación, como el acceso o la cobertura, y, a partir de ahí, encontrarle un sentido a reunirnos dos veces por semana a intentar transmitir o adquirir ciertos saberes. Si tuviera que concluir algo con respecto a mi experiencia de práctica, diría que me parece importante transmitirles a las y los estudiantes el sentido de un tipo de sistema educacional y las razones por las cuales conversamos, discutimos y debatimos ciertos temas. En función de la situación actual que vivimos en el país y en el mundo, me parece necesario transmitir de alguna forma que tenemos la posibilidad de repensar absolutamente todas nuestras acciones y cómo ellas influyen en la organización social. De este modo, creo que es menester de las y los docentes enfrentar las reflexiones y los debates que se puedan generar en la escuela con respecto a lo anteriormente dicho.

En el documento Marco para la Buena Enseñanza del Ministerio de Educación del Estado de Chile, se mencionan cuatro dominios que se deben asegurar para la transmisión de saberes: la preparación de la enseñanza, la creación de un ambiente propicio para la enseñanza, la enseñanza para el aprendizaje de todas y todos los estudiantes, y las responsabilidades profesionales implicadas en el proceso de enseñanza. Más allá de las buenas intenciones, habría que preguntarse si en el contexto actual de precarización del sistema de enseñanza en general es posible, efectivamente, asegurar estos cuatro dominios. Deberíamos preguntarnos si existen razones por las que un sistema educacional está en constante cambio, como si se estuviera haciendo y rehaciendo constantemente, con supuestos objetivos sociales, con supuestas motivaciones “modernizadoras". Se puede entender que hemos llegado a un momento en que el sistema educacional está principalmente en manos de privados, como es el caso de la fundación en la que realicé la práctica profesional, y que se ha generado una desvalorización continua de la educación. La creación del mercado educacional y la pérdida de control por parte del Estado revelan que los conceptos de "mercado" y "calidad" son evidentemente segregadores, atomizadores y perpetuadores de un sistema injusto. En este marco, entonces, la cobertura puede llegar a perder toda importancia y también lo puede hacer el sentido fundamental de la educación, en la medida que, en términos socioeconómicos, no nos hemos preguntado cuáles son los reales objetivos que debería perseguir un sistema educacional. Lo anterior pareciera ser algo fundamental para poder establecer las bases sobre las que construimos un sistema educacional determinado. Sin embargo, sin hacer primero aquella reflexión y al ir siempre organizando el sistema sobre la marcha, tenemos entonces el sistema educacional actual, controlado principalmente por grupos económicos que no 
tienen ninguna intención de ayudar a los/as estudiantes a desarrollarse, formarse ni constituirse en sujetos críticas y críticos. Cezar Migliorin (2018) lo expresa de esta manera:

En cuanto a la privatización de la educación, sabemos que no sucede solo cuando es el lucro lo que la organiza, sino cuando lo que sucede en la escuela tiene como objetivo la formación de personal para las empresas y corporaciones que tienen al lucro como opción central, o cuando la competencia se naturaliza entre docentes y estudiantes. El pragmatismo de este modelo niega que cualquier invención de mundo pueda atravesar la escuela, y esta es la más cruel de las privatizaciones; una apropiación de la escuela como productora de mano de obra y de consumidores para la reproducción de un orden del capital a expensas de lo que es común, independientemente de clase. (pp.195-196)

El Marco para la Buena Enseñanza persigue objetivos que parecieran ser un puñado de buenas intenciones, las cuales no se condicen necesariamente con la realidad de la educación. Pareciera, entonces, ser una tarea de los actores y actoras educativos/as instalar, como ya se ha dicho, las discusiones con respecto al sentido de educarnos y al lugar que debe ocupar la educación en nuestra sociedad. Es en este contexto que el Simce aparece como una temática importante no solo para esta fundación, sino para todo el sistema educacional, pues, como señala Bellei (2020), "la lógica fundacional del Simce fue que, para hacer que el mercado funcionara con información válida, había que entregar a las familias un indicador de la calidad de las ofertas educacionales, para que eligieran racionalmente" (p. 82). Este autor expresa, además, que desde que comenzaron a publicarse los resultados de las pruebas de medición estandarizadas, la autoridad educacional ha fomentado que las familias puedan escoger los establecimientos basándose en los puntajes obtenidos, "llegando a enviar cartas personales a los padres con las escuelas convertidas en luces de semáforo, para que nadie se confundiera llevando a sus hijos a escuelas rojas y todos pelearan por un cupo en las verdes" (Bellei, 2020, p.82).

Por otro lado, es necesario reflexionar también con respecto a los saberes que están siendo transmitidos en los colegios y cómo se está llevando a cabo el proceso de enseñanza-aprendizaje, ya no solo en términos de educación -de emergencia- a distancia, mediante clases sincrónicas o asincrónicas, sino también en términos de cómo se están acercando ciertos saberes, dejando premeditadamente otros de lado. Toda selección de saberes se produce en una realidad cultural determinada y, así, la transmisión de conocimientos está necesariamente determinada por la cultura. 
¿Cómo entendemos el concepto "cultura"?: "Podría decirse que todo producto o práctica humana es cultural, ya que toda acción u objeto siempre es experimentado dentro de un universo de significados producidos por el hombre" (Gvirtz y Palamidessi, 2006, p.21). Si la selección de ciertos saberes tiene que ver con su validación (ya sea institucional o social) y se entiende a el y la docente como un/a agente activo/a en el proceso de transmisión de conocimientos (aprendizajes), entonces se podría decir que el rol de los profesores y las profesoras implica la validación de aquellos saberes que corresponderán a los contenidos enseñados. Giroux lo explica de la siguiente manera:

[en] el sentido más amplio, los profesores como intelectuales han de contemplarse en función de los intereses ideológicos y políticos que estructuran la naturaleza del discurso, las relaciones sociales de aula y los valores que ellos mismos legitiman en su enseñanza. (1997, p.65)

Se debe considerar, además, que "el contenido a enseñar es, en buena medida, algo relativamente indeterminado; está parcialmente abierto y debe ser materializado a través de la enseñanza" (Gvirtz y Palamidessi, 2006, p.19).

\section{Referencias}

Bellei, C. (2020). Educación para el siglo XXI en el siglo XXI ¿Tomamos el tren correcto? Horizontes y propuestas para transformar el sistema educativo chileno. Biblioteca del Congreso Nacional de Chile.

Dolz, J. (1994). La interacción de las actividades orales y escritas en la enseñanza de la argumentación. Comunicación, Lenguaje y Educación, 6(3), 17-27. https://doi.org/10.1174/021470394321466855

Giroux, H. (1997). Los profesores como intelectuales: hacia una pedagogía crítica del aprendizaje. Paidós. 
Gvirtz, S. y Palamidessi, M. (2006). El abc de la tarea docente: currículum y enseñanza. Editorial Aique.

Fairclough, N. (2003). El análisis crítico del discurso como método para la investigación en ciencias sociales. En R. Wodak y M. Meyer (Comps.), Métodos de análisis crítico del discurso (Pp. 179- 203). Gedisa.

Lorenz, F. (2004). “Tomála vos, dámela a mí". La noche de los lápices: el deber de recordar y las escuelas. En E. Jelin y F. Lorenz (Comps.), Educación y memoria (pp. 95-129). Siglo XXI.

Magendzo, A. (2003). Pedagogía crítica y educación en derechos humanos. Paulo Freire. Revista de Pedagogía Crítica, 2(2),19-27.

Migliorin, C. (2018). Pedagogía del lío. Cine, educación y política. Cuarto Propio.

Ministerio de Educación (2008). Marco para la Buena Enseñanza. https://www.docentemas.cl/docs/MBE2008.pdf

Ministerio de Cultura y Educación (1977). Subversión en el ámbito educativo (conozcamos a nuestros enemigos). http://repositorio.educacion.gov.ar/dspace/handle/123456789/88827

Rockwell, E. (1997). La escuela cotidiana. Fondo de Cultura Económica.

Sábato, E. (1998). Querido y remoto muchacho. Losada.

Scolari, C. (2014). Narrativas transmedia: nuevas formas de comunicar en la era digital. Anuario AC/E de cultura. Acción Cultural Española. https://www.accioncultural.es/media/Default\%20Files/activ/2014/Adj/Anuario_ACE_20 14/6Transmedia_CScolari.pdf 